\section{Exogenous Reference RNA for Normalization of Real-Time Quantitative PCR}

BioTechniques 34:88-91 (January 2003)

\begin{abstract}
We have utilized an in vitro transcribed $3^{\prime}$ mRNA fragment of the plant gene ribulose bisphosphate carboxylase (RuBisCO) as an exogenous standard for normalization of quantitative PCR data. Both K562 cells and primary erythroid $\mathrm{CD} 34^{+}$progenitor cells were treated with sodium butyrate and changes in $\gamma$-globin mRNA levels were assayed using a previously published TaqMan ${ }^{\circledR}$ probe and primer set, while RuBisCO levels were assayed by a SYBR ${ }^{\circledR}$ Green detection assay. The data presented show that a correction to measured $\gamma$-globin induction was necessary with both cell types. The correction for the $C D 34^{+}$progenitor cells was a striking 95\% increase, while that for the K562 cells was 44\%. The use of an exogenous reference such as in vitro transcribed mRNA for the RuBisCO plant gene provides a robust and sample-independent method for the normalization of quantitative PCR data in bacterial and animal cells.
\end{abstract}

\section{INTRODUCTION}

The increasingly widespread use of fluorescence-based, real-time, quantitative PCR has provided many researchers with the ability to determine precisely levels of a target nucleic acid within a given tissue or cell sample as opposed to a mere qualitative demonstration of its presence or absence or even semi-quantitative characterization by classical PCR methods. Indeed, we have demonstrated the absolute quantitation of gene expression in single cells using this technique (10), while others have demonstrated single copy template detection (15). The TaqMan ${ }^{\circledR}$ assay represents one of the earliest and more common implementations of the technology and involves the inclusion of a dual fluorescently labeled oligonucleotide probe within the PCR, with the $3^{\prime}$ fluorophore acting as a quencher of the emission of the $5^{\prime}$ fluorophore. The $5^{\prime}$ nuclease activity of Taq DNA polymerase causes the spatial separation of both labels producing one active fluorophore for each new strand of cDNA synthesized during PCR (6). Other formats have been developed including intercalating fluorescent dyes (15), molecular beacons (12), and hybridization probes (15), with new ones being introduced currently. Some formats have proven more suitable for specific applications, such as allelic discrimination or determination of PCR product melting points as an indication of the reaction's specificity.

Currently, real-time quantitative PCR is often used to assess changes or differences in gene expression (as indicated by mRNA levels) between different biological samples. With few exceptions, the thermostable DNA polymerases commonly used in PCR require DNA as template for amplification. This necessitates the generation of complementary DNA templates from mRNA by reverse transcription before performing the PCR for the desired target. This is commonly accomplished using reverse transcriptase either from Moloney murine leukemia virus or avian myeloblastosis virus. This reverse transcription step has been proposed as the source of most of the variability in quantitative RT-PCR experiments (4), owing to the sensitivity of reverse transcriptase enzymes to salts, aliphatic alcohols, and phenol commonly used during the RNA isolation step. Nucleic acid templates extracted from a variety of biological samples have also been shown to contain inhibitors of the enzymes used in either reverse transcription or PCR or both $(5,7,8)$. This DNA polymerase inhibition, along with the variations in nucleic acid extraction efficiency, can lead to misinterpretation of the expression levels of target sequences.

Attempts are made to correct for such errors by normalizing the levels of the target mRNA to that of an internal reference nucleic acid, of which $\beta$-actin, GAPDH, or rRNAs are among the most commonly used. Ideally, an internal reference RNA should maintain a constant expression level across tissues and during development, as well as between in- dividuals. The commonly used internal reference RNAs unfortunately are found lacking in one or more of the criteria mentioned above $(11,16)$. Hence, the internal reference for normalization in any given experiment must be carefully considered and screened for consistency. It is mainly for these reasons that, previously, the metric that we have usually used in this laboratory was to normalize all copy numbers or target mRNA concentrations to the total RNA concentration $(9,10)$.

As an alternative to an endogenous RNA reference, we have explored the possibility of using an exogenous reference mRNA produced by in vitro transcription for the purpose of normalizing target nucleic acid levels determined by real-time quantitative PCR. We chose the commercially available ribulose bisphosphate carboxylase $(\mathrm{Ru}-$ BisCO) plant RNA, which codes for an enzyme that catalyzes the combination of carbon dioxide with ribulose 1,5-bisphosphate to produce two molecules of 3-phosphoglycerate and represents the first step in carbon fixation by plants. This gene is not known to occur in animals, so the addition of a fixed amount to each cell or tissue lysate from which nucleic acid is to be extracted can provide a reference template within each sample for the purpose of normalizing the levels of the target nucleic acid.

We report here the assay of $\gamma$-globin levels in the erythroleukemic K562 cell line as well as primary CD $34^{+}$cells by real-time quantitative PCR in response to induction by $1 \mathrm{mM}$ sodium butyrate and the amplification of an exogenous RuBisCO template with a $\mathrm{SYBR}^{\circledR}$ Green detection method (Molecular Probes, Eugene, OR, USA) to control for variation resulting from the inhibition of DNA polymerase activity both in reverse transcription and template amplification by PCR.

\section{MATERIALS AND METHODS}

\section{Cell Culture}

Erythroleukemic K562 cells were obtained from ATCC (Manassas, VA, USA) and grown in RPMI 1640 culture medium supplemented with 10\% FCS (Intergen, Purchase, NY, USA), 100 
$\mathrm{U} / \mathrm{mL}$ penicillin, and $100 \mu \mathrm{g} / \mathrm{mL}$ streptomycin (Biofluids, Rockville, MD, USA). Primary CD34+ cells were isolated by immunomagnetic separation from cultures of peripheral blood mononuclear cells that were obtained with informed consent from normal donors. Peripheral blood mononuclear cells were prepared by centrifugation on a cushion of Lymphocyte Separation Medium (ICN Biomedicals, Costa, Mesa, CA, USA), washed twice with Dulbecco's PBS (Invitrogen, Carlsbad, CA, USA), and resuspended in $\alpha$-minimum essential medium (Sigma, St. Louis, MO, USA) supplemented with $10 \%$ FCS, $1 \mu \mathrm{g} / \mathrm{mL}$ cyclosporin A (Sandoz, Basel, Switzerland), $2 \mathrm{mM}$ glutamine, $100 \mathrm{U} / \mathrm{mL}$ penicillin, 100 $\mu \mathrm{g} / \mathrm{mL}$ streptomycin (all from Biofluids), and $10 \%$ conditioned medium obtained from cultures of the 5637 bladder carcinoma cell line. After one week, the non-adherent cells were har- vested from this phase I culture by centrifugation, washed twice in $\alpha$-medium (without supplements), and resuspended in fresh $\alpha$-medium with $30 \%$ FCS, 2 $\mathrm{mM}$ glutamine, $100 \mathrm{U} / \mathrm{mL}$ penicillin, $100 \mu \mathrm{g} / \mathrm{mL}$ streptomycin, $10 \%$ deionized BSA, $10^{-5} \mathrm{M} \beta$-mercaptoethanol, $10^{-6} \mathrm{M}$ dexamethasone, $33 \mu \mathrm{g} / \mathrm{mL}$ holo-transferrin (Sigma), and $1 \mathrm{U} / \mathrm{mL}$ human recombinant erythropoietin (Ortho Pharmaceutical, Raritan, NJ, USA). Cultures were incubated at $37^{\circ} \mathrm{C}$ in an atmosphere of $5 \% \mathrm{CO}_{2}$ in air with extra humidity. Sodium butyrate from a $1 \mathrm{M}$ stock in base medium was added to a final concentration of $1 \mathrm{mM}$, and cells were incubated for a further $48 \mathrm{~h}$.

Total RNA was extracted from approximately $10^{6}$ cells using the RNeasy ${ }^{\circledR}$ mini kit (Qiagen, Valencia, $\mathrm{CA}, \mathrm{USA}$ ). The in vitro transcribed $\mathrm{Ru}-$ BisCO mRNA (Stratagene, La Jolla, CA, USA) was added ( $1 \mathrm{ng})$ to the RLT cell lysis buffer. The extracted RNA was

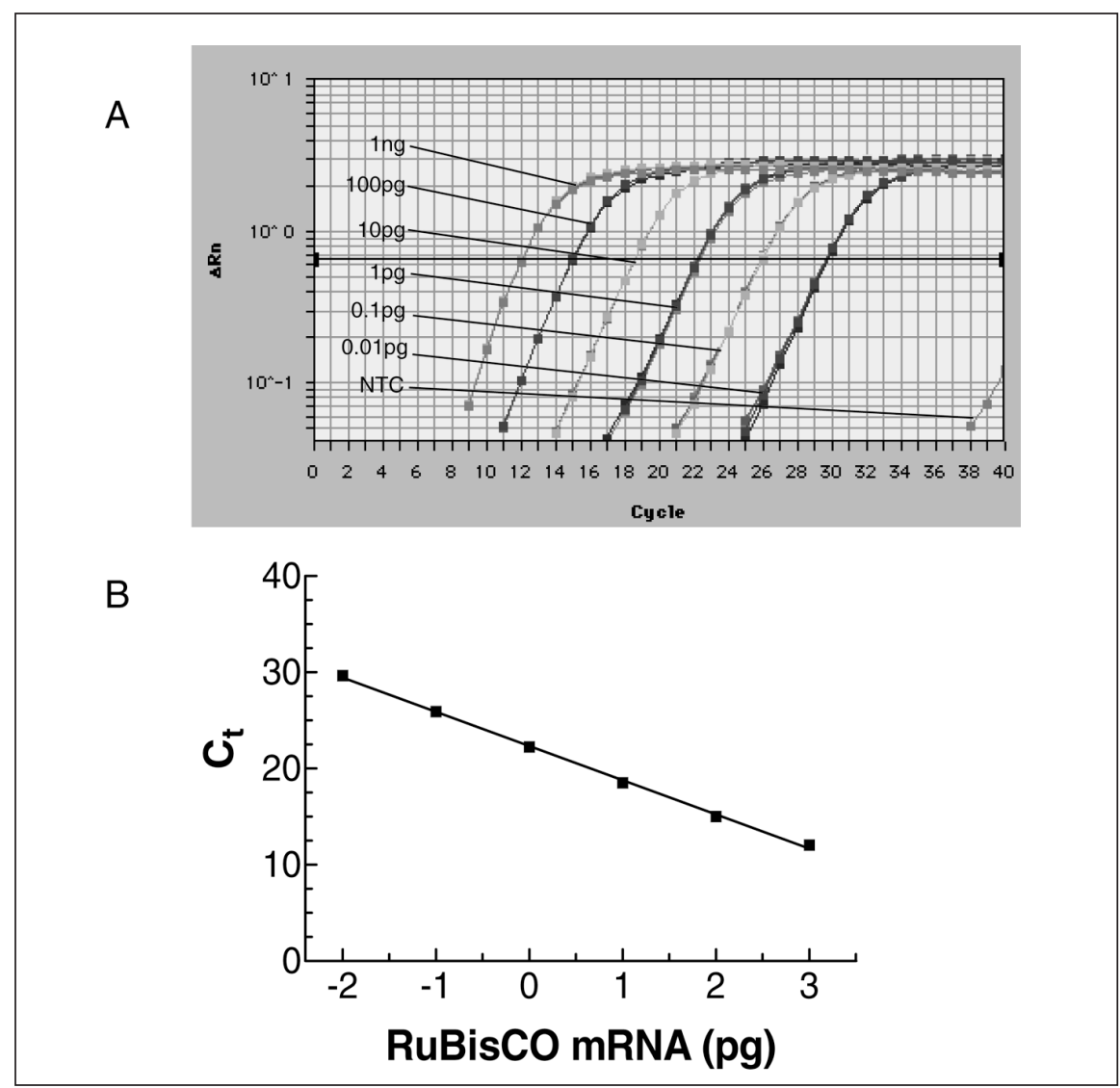

Figure 1. Linear dose response of RuBisCO mRNA template titration. RuBisCO mRNA was reverse-transcribed to produce cDNA and assayed in quadruplicate by quantitative PCR. A $10^{5}$-fold range of mRNA template was used ( $10 \mathrm{fg}$ to $\left.1 \mathrm{ng}, \mathrm{R}^{2}=0.999\right)$. The data shown represent typical results obtained from one of three experiments. quantified by spectrophotometry and integrity confirmed by electrophoresis.

\section{Real-Time Quantitative PCR}

RNA $(1 \mu \mathrm{g})$ was reverse-transcribed using an enzyme mixture that consisted of $4 \mu \mathrm{L} 5 \times$ RT buffer, $10 \mathrm{mM}$ DTT, 0.5 mM dNTP mixture, $0.5 \mu \mathrm{g}$ oligo $(\mathrm{dT})_{24}$, $1 \mu \mathrm{L}$ RNAseout, and $200 \mathrm{U}$ SuperScript $^{\mathrm{TM}}$ II (Invitrogen) in DEPC-treated water. The tubes were incubated at $42^{\circ} \mathrm{C}$ for $60 \mathrm{~min}$, followed by $10 \mathrm{~min}$ at $80^{\circ} \mathrm{C}$ to inactivate the enzyme.

Real-time quantitative PCR assay of globin transcripts was carried out using gene-specific double fluorescently labeled probes in a 7700 Sequence Detector (Applied Biosystems, Foster City, CA, USA). All probes are designed to span exon junctions in the fully processed message to prevent reporting of amplification of any possible contaminating genomic DNA. All primers and probes were made using reagents from Glen Research (Chantilly, VA, USA) on an ABI 394 synthesizer (Applied Biosystems). 6-Carboxy fluorescein (FAM) was used as the $5^{\prime}$ fluorescent reporter, while tetramethylrhodamine (TAMRA) was added to the $3^{\prime}$ end as quencher. Probe and primers used for quantitation of $\gamma$-globin have been previously reported (9). Double fluorescently labeled (TaqMan) probes were HPLCpurified on a $250 \times 10 \mathrm{~mm}$ Biovantage C8 reverse phase column (Thomson, Chantilly, VA, USA) using a $10 \%-35 \%$ acetonitrile gradient in $0.1 \mathrm{M}$ triethylamine acetate, $\mathrm{pH} 7.0$, with the detector set to monitor column eluate at 260, 494, and $565 \mathrm{~nm}$. Products were lyophilized and characterized by absorption spectroscopy as well as DNase I treatment to confirm a greater than or equal to 2-fold increase in fluorescence. The following primers were used for RuBisCO assay: forward, 5'-GAAGAAGCGGGGATACTTGGTC-3', and reverse, 5'-AGTGTTGTGTCCACAAGAAATCC-3'. SYBR Green was used as reporter for real-time PCR using RuBisCO primers. All oligonucleotide primers and probes were quantitated by absorbance at 260 nm. Specific transcripts within cDNA reaction products were quantitated in a reaction mixture consisting of $10 \mathrm{mM}$ Tris, $\mathrm{pH} 8.3,50 \mathrm{mM} \mathrm{KCl}, 3 \mathrm{mM} \mathrm{MgCl}$, $200 \mu \mathrm{M}$ dNTP (dTTP replaced with 
dUTP at $400 \mu \mathrm{M}), 0.01 \mathrm{U} / \mu \mathrm{L}$ uracil DNA glycosylase (Roche Applied Science, Indianapolis, IN, USA), and 0.025 U/ $\mu$ L Platinum Taq DNA polymerase (Invitrogen). Standard curves were constructed using dilutions of an accurately determined plasmid containing the cDNA of interest as template. A dynamic range of $5 \log$ orders of concentration or greater was routinely achieved for each transcript of interest.

\section{RESULTS AND DISCUSSION}

One requirement of a standard template for quantitative PCR is a linear response over the expected range of concentration in samples to be determined. Figure 1 demonstrates such a linear response for dilutions of RuBisCO template over five orders of magnitude with each dilution being assayed in quadruplicate. The advent of PCR has resulted in a quantum leap in the sensitivity of techniques used to detect gene expression at the transcript level in cells and tissue specimens but was not easily quantitated. The introduction of competitive PCR $(13,14)$ facilitated more accurate determination of changes in levels of gene expression in physiological and pharmacological systems. However, the process was laborious and time consuming and was not amenable to analysis of large numbers of samples, mainly because of the requirement for the construction of the competing template for each new transcript of interest and the extensive post-PCR analysis required, involving gel electrophoresis, image acquisition, and densitometry The use of the more recently introduced real-time, fluorescence-based quantitative PCR is rapidly growing and promises to be an invaluable tool in the analysis of gene expression, especially when high-throughput analysis is required. Even with the greater ease of use that real-time quantitative PCR has provided the researcher, normalization of gene expression data still remains an important aspect of accurate determination and is still routinely done using levels of an endogenous transcript. Several reports have been published highlighting the drawbacks of using an endogenous transcript such as that of a housekeeping gene $(2,11,16)$. It is now well known that the expression level of genes such as $\beta$-actin and GAPDH varies from tissue to tissue and from one cell type to another. In addition, transcript levels from these genes vary considerably in response to a great variety of pharmacologic interventions and disease states (2). Such variability in the levels of reference or housekeeping genes may not parallel or be analogous to concomitant changes in the levels of a particular gene of interest, making the normalization of gene expression data

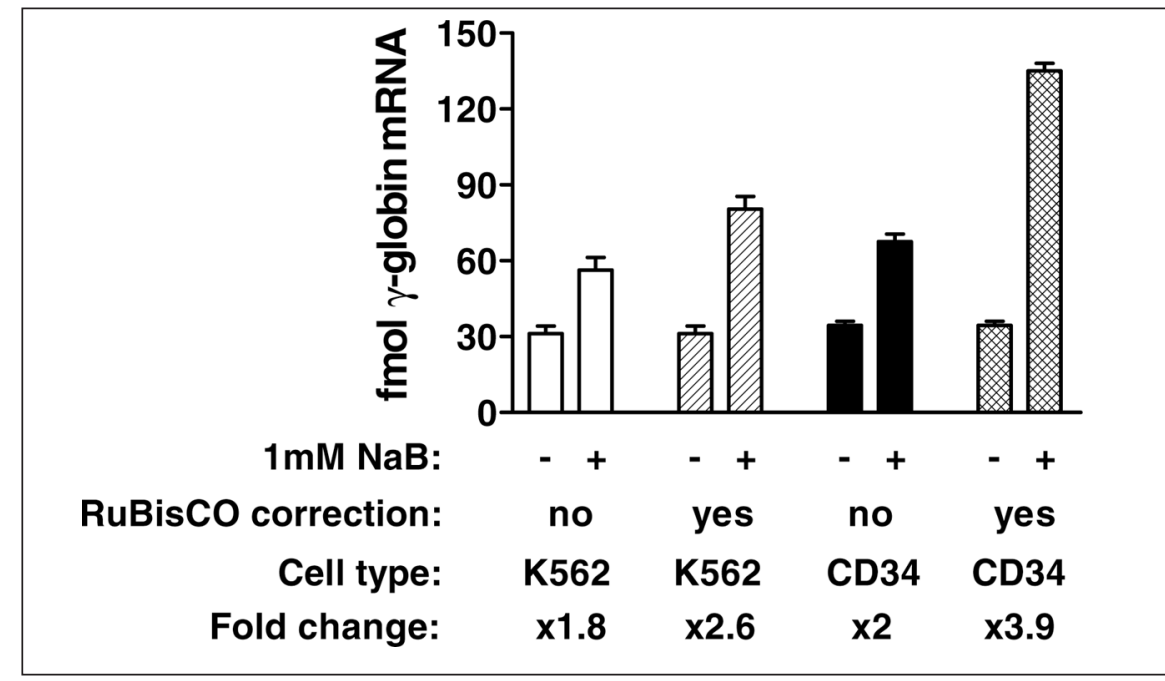

Figure 2. Absolute amounts of $\gamma$-globin mRNA in $1 \mu$ g total (K562 or CD34 progenitor) cellular RNA with and without $1 \mathrm{mM}$ sodium butyrate for $48 \mathrm{~h}$. Results shown represent amounts of $\gamma$-globin mRNA template before and after correction based on levels of exogenous RuBisCO reference mRNA template levels. Samples were assayed in triplicate and represent one of two experiments with similar results. between samples problematic and error prone. Interestingly, a recent publication (3) has proposed the use of peptidylprolyl isomerase A as a preferred internal reference over GAPDH and $\beta$ actin in quantitative PCR analyses after screening thousands of genes in a variety of human tissue types. While it remains to be seen if this will indeed be the case, the continuing search for an appropriate internal reference further highlights the need for a sample-independent exogenous reference.

In Figure 2, we have shown that by adding a fixed amount of in vitro transcribed, polyadenylated RuBisCO transcript to the lysate for each sample to be analyzed by real-time quantitative PCR, one can control for the presence of inhibitors of both reverse transcription and PCR DNA polymerases. The fold change in $\gamma$-globin induction in K562 cells by sodium butyrate was corrected from 1.8 -fold to 2.6 -fold (a $44 \%$ increase), while that for $\mathrm{CD} 34^{+}$progenitor cells was corrected from 2-fold to 3.9fold (a 95\% increase) based on this external standard. While the necessity of such corrections has been long identified, a robust and standard method of correction has proved difficult to establish. As an alternative to the use of an endogenous transcript, the addition of a purified and well-characterized control template, such as the in vitro transcribed $\mathrm{RuBisCO}$ template used in this study, at the stage of RNA template isolation should provide a valid method of quantitative PCR data normalization. At present, we are aware of one other publication (1) that uses a similar rationale to examine changes in the level of prostaglandin D synthetase mRNA during development in mouse testis with luciferase mRNA as an exogenous reference. Of course, an alternative reference template will have to be implemented by investigators wishing to perform quantitative PCR studies on plant material, but this general method should be of value to those studying any of the several other kingdoms of the living world.

\section{REFERENCES}

1.Baker, P.J. and P.J. O'Shaughnessy. 2001. Expression of prostaglandin D synthetase during development in the mouse testis. Repro- 
duction 122:553-559.

2.Bustin, S.A. 2000. Absolute quantification of mRNA using real-time reverse transcription polymerase chain reaction assays. J. Mol. Endocrinol. 25:169-193.

3.Feroze-Merzoug, F., I.M. Berquin, J. Dey, and Y.Q. Chen. 2002. Peptidylprolyl isomerase A (PPIA) as a preferred internal control over GAPDH and $\beta$-actin in quantitative RNA analyses. BioTechniques 32:776-782.

4.Freeman, W.M., S.J. Walker, and K.E. Vrana. 1999. Quantitative RT-PCR: pitfalls and potential. BioTechniques 26:112-115.

5.Khan, G., H.O. Kangro, P.J. Coates, and R.B. Heath. 1991. Inhibitory effects of urine on the polymerase chain reaction for cytomegalovirus DNA. J. Clin. Pathol. 44:360365 .

6.Livak, K.J., S.J. Flood, J. Marmaro, W. Giusti, and K. Deetz. 1995. Oligonucleotides with fluorescent dyes at opposite ends provide a quenched probe system useful for detecting PCR product and nucleic acid hybridization. PCR Methods Appl. 4:357-362.

7.Makowski, G.S., E.L. Davis, J. Aslanzadeh, and S.M. Hopfer. 1995. Enhanced direct amplification of Guthrie card DNA following selective elution of PCR inhibitors. Nucleic Acids Res. 23:3788-3789.

8.Poddar, S.K., M.H. Sawyer, and J.D. Con- nor. 1998. Effect of inhibitors in clinical specimens on Taq and Tth DNA polymerase-based PCR amplification of influenza A virus. J. Med. Microbiol. 47:1131-1135.

9.Smith, R.D., J. Li, C.T. Noguchi, and A.N. Schechter. 2000. Quantitative PCR analysis of $\mathrm{HbF}$ inducers in primary human adult erythroid cells. Blood 95:863-869.

10.Smith, R.D., J.D. Malley, and A.N. Schechter. 2000. Quantitative analysis of globin gene induction in single human erythroleukemic cells. Nucleic Acids Res. 28:4998-5004.

11.Thellin, O., W. Zorzi, B. Lakaye, B. De Borman, B. Coumans, G. Hennen, T. Grisar, A Igout, and E. Heinen. 1999. Housekeeping genes as internal standards: use and limits. J. Biotechnol. 75:291-295.

12.Tyagi, S. and F.R. Kramer. 1996. Molecular beacons: probes that fluoresce upon hybridization. Nat. Biotechnol. 14:303-308.

13. Vanden Heuvel, J.P., F.L. Tyson, and D.A Bell. 1993. Construction of recombinant RNA templates for use as internal standards in quantitative RT-PCR. BioTechniques 14:395398.

14.Wang, A.M., M.V. Doyle, and D.F. Mark. 1989. Quantitation of mRNA by the polymerase chain reaction. Proc. Natl. Acad. Sci. USA 86:9717-9721.
15.Wittwer, C.T., M.G. Herrmann, A.A. Moss, and R.P. Rasmussen. 1997. Continuous fluorescence monitoring of rapid cycle DNA amplification. BioTechniques 22:130-138

16.Zhong, H. and J.W. Simons. 1999. Direct comparison of GAPDH, $\beta$-actin, cyclophilin, and 28S rRNA as internal standards for quantifying RNA levels under hypoxia. Biochem. Biophys. Res. Commun. 259:523-526.

Address correspondence to Dr. Reginald Smith, Laboratory of Chemical Biology, NIDDK, NIH, Building 10, Room 9N318, 10 Center Drive, MSC 1822, Bethesda, MD 20892-1822,USA. e-mail: smithrd@helix. nih.gov

Received 1 May 2002; accepted 21 October 2002.

Reginald D. Smith, Benjamin Brown, Pranvera Ikonomi, and Alan N. Schechter National Institutes of Health Bethesda, MD, USA 